\title{
Sodium hydrosulfide alleviates dexamethasone-induced cell senescence and dysfunction through targeting the miR-22/sirt1 pathway in osteoblastic MC3T3-E1 cells
}

\author{
PENG LI ${ }^{1}$, WEI-WEI MAO ${ }^{2}$, SHUAI ZHANG ${ }^{1}$, LIANG ZHANG ${ }^{1}$, ZHI-RONG CHEN $^{1}$ and ZHI-DONG LU ${ }^{1}$ \\ ${ }^{1}$ Department of Orthopedics, General Hospital of Ningxia Medical University, Xingqing, Yinchuan, Ningxia 750004; \\ ${ }^{2}$ Clinical Skill Center of Yinchuan First People's Hospital, Yinchuan, Ningxia 750001, P.R. China
}

Received February 20, 2020; Accepted September 15, 2020

DOI: $10.3892 / \mathrm{etm} .2021 .9669$

\begin{abstract}
Glucocorticoid-induced osteoporosis is characterized by osteoblastic cell and microarchitecture dysfunction, as well as a loss of bone mass. Cell senescence contributes to the pathological process of osteoporosis and sodium hydrosulfide (NaHS) regulates the potent protective effects through delaying cell senescence. The aim of the present study was to investigate whether senescence could contribute to dexamethasone (Dex)-induced osteoblast impairment and to examine the effect of NaHS on Dex-induced cell senescence and damage. It was found that the levels of the senescence-associated markers, p53 and p21, were markedly increased in osteoblasts exposed to Dex. A p53 inhibitor reversed Dex-induced osteoblast injury, a process that was mitigated by NaHS administration through alleviating osteoblastic cell senescence. MicroRNA (miR)-22 blocked the impact of NaHS on Dex-induced osteoblast damage and senescence through targeting the regulation of Sirtuin 1 (sirt1) expression, as shown by the decreased cell viability and alkaline phosphatase activity, as well as an increased expression of p53 and p21. It was revealed that the sirt1 gene was the target of miR-22 in osteoblastic MC3T3-E1 cells through combining the results of dual luciferase reporter assays and reverse transcription-quantitative PCR, as well as western blot analyses. Silencing of sirt1 abolished the protective effect of NaHS against Dex-associated osteoblast senescence and injury. Taken together, the present study showed that NaHS prevents Dex-induced cell senescence and damage through targeting the miR-22/sirt1 pathway in osteoblastic MC3T3-E1 cells.
\end{abstract}

Correspondence to: Professor Zhi-Dong Lu, Department of Orthopedics, General Hospital of Ningxia Medical University, 804 Shengli South Road, Xingqing, Yinchuan, Ningxia 750004, P.R. China

E-mail: zhidonglu@126.com

Key words: sodium hydrosulfide, senescence, microRNA-22, Sirtuin 1, osteoblasts, dexamethasone

\section{Introduction}

Glucocorticoids (GCs) are used clinically to treat a number of diseases, and it is estimated that $1-2 \%$ of the worldwide population are undergoing long-term GC therapy (1-4). However, long-term administration with GCs has been shown to result in multiple complications, common among which is GC-induced osteoporosis (GIO), which is characterized by systemic damage of the bone size and microarchitecture (5). A growing number of studies have revealed that osteoblast dysfunction disrupts the balance between bone formation and bone resorption in terms of the role that is exerted by bone mass in GIO, for example, by inhibiting osteoblast proliferation and differentiation, as well as enhancing the rate of osteoblast apoptosis (5-7).

Cell senescence is considered to be a state of cell cycle arrest, as well as physiological and metabolic dysfunction, which occurs under normal environmental conditions, with a gradual increase in its prevalence towards death (8). A previous study showed that cell senescence serves an important role in the development of osteoporosis (9). However, whether osteoblast senescence contributes to the development of GIO has yet to be elucidated.

Hydrogen sulfide $\left(\mathrm{H}_{2} \mathrm{~S}\right)$ is an endogenously generated secondary messenger that is involved in regulating a wide range of physiological processes, including having cardioprotective and neuroprotective effects, as well exerting a beneficial role following acute lung injury (10-12). Furthermore, $\mathrm{H}_{2} \mathrm{~S}$ also has a critical role in the occurrence and development of osteoporosis $(13,14)$. Xu et al (13) demonstrated that $\mathrm{H}_{2} \mathrm{~S}$ attenuates oxidative stress-induced osteoblastic cell damage and inhibits cell proliferation, as well as differentiation, through a MAPK-dependent signaling pathway. Additionally, Yang et al (14) found that $\mathrm{H}_{2} \mathrm{~S}$ mitigates dexamethasone (Dex)-induced osteoblast injury by activating the AMP-activated protein kinase signaling (14); however, whether $\mathrm{H}_{2} \mathrm{~S}$ inhibits Dex-induced osteoblast dysfunction through repressing cell senescence has yet to be elucidated.

MicroRNAs (miRs), as a class of highly conserved noncoding RNAs, which are able to post-transcriptionally modulate the expression of target genes and serve a critical role in various diseases (15-17). Numerous studies have shown 
that miRs contribute to the pathogenesis and development of osteoporosis (18-20). Notably, previous research showed that miR-22 was negatively correlated with the function of various cells $(17,21,22)$. In the present study, alterations in the expression profile of biomarkers, such as p53 and p21, which are associated with cell senescence in Dex-treated MC3T3-E1 cells, and the effects of sodium hydrosulfide (NaHS) on Dex-induced osteoblast damage and senescence, were examined. Subsequently, the expression levels of miR-22 in Dex-treated osteoblast cells with or without NaHS administration were investigated to determine whether miR-22 is involved with the protective role that NaHS has in Dex-induced osteoblast dysfunction and cell senescence.

\section{Materials and methods}

Cell culture and drug administration. Osteoblastic murine MC3T3-E1 cells were cultured in $\alpha$-MEM ( $\alpha$-MEM, Gibco; Thermo Fisher Scientific, Inc.) with $10 \%$ FBS (Thermo Fisher Scientific, Inc.) at a temperature of $37^{\circ} \mathrm{C}$ and in an atmosphere containing 5\% $\mathrm{CO}_{2} / 95 \%$ air. Absolute ethanol was used to dissolve the Dex (Sigma-Aldrich; Merck KGaA) and the cells were treated with Dex at a final concentration of $1 \mu \mathrm{M}$ for $72 \mathrm{~h}$, as previously described (23). The p53 inhibitor, pifithrin- $\alpha$ (Sigma-Aldrich; Merck KGaA) was dissolved in DMSO and the cells were treated with pifithrin- $\alpha$ at a concentration of $20 \mu \mathrm{M}$ for $48 \mathrm{~h}$ (23). NaHS (Sigma-Aldrich; Merck KGaA) was dissolved in saline solution and used at $20 \mu \mathrm{M}$ for $72 \mathrm{~h}$. Dex and NaHS after 24 h of cell culture, and pifithrin- $\alpha$ was used after $48 \mathrm{~h}$. The cell number used in these experiments was $2 \times 10^{5}$ cells $/ \mathrm{ml}(200 \mu \mathrm{l}$ each well in 48 -well plate and $2 \mathrm{ml}$ each well in 6-well plate).

Small interfering (si)RNA transfection assay. Xfect ${ }^{\mathrm{TM}}$ RNA transfection reagent (Takara Biotechnology Co., Ltd.) was used to perform sirtuin 1 (sirtl) siRNA transfections $(200 \mathrm{nM}$; cat. no. sc-40987; Santa Cruz Biotechnology, Inc.) into osteoblastic MC3T3-E1 cells, with a non-targeting siRNA (Santa Cruz Biotechnology, Inc.) served as negative control. The sequences of control siRNA are as follows: 5'-UUCUUC GAACGUGUCACGUTT-3' and 5'-ACGUGACACGUUCGG AGAATT-3'. Osteoblasts were pretreated with control or sirt1 siRNA for $24 \mathrm{~h}$ and then immediately subjected to subsequent experimentation.

Cell viability analysis. MTT detection assays were performed to measure the viability of the osteoblasts. Briefly, osteoblastic cells were cultivated in a 48 -well plate at a density of $2 \times 10^{5}$ cells $/ \mathrm{ml}$ (200 $\mu \mathrm{l}$ each well) at $37^{\circ} \mathrm{C}$ and the cells were subjected to Dex and NaHS treatment for $72 \mathrm{~h}$, and pifithrin- $\alpha$. was added for the last $48 \mathrm{~h}$. Cells were then exposed to MTT for $2-4 \mathrm{~h}$ at $37^{\circ} \mathrm{C}$ in the dark and a microplate reader was used to detect absorbance of formazan crystals dissolved in DMSO at $550 \mathrm{~nm}$.

Alkaline phosphatase (ALP) activity detection. The method described by Bowers and McComb was performed to measure ALP activity (24). Briefly, Triton X-100 was added into $1 \times 10^{5}$ osteoblasts at a final concentration of $1 \%$. The supernatant was collected by centrifugation at $14,000 \mathrm{x}$ g at $4^{\circ} \mathrm{C}$ for $20 \mathrm{~min}$. A total of $25 \mu \mathrm{l}$ supernatant was added into the reaction mixture, which contained assay buffer and para-nitrophenyl phosphate (cat. no. P0321S; Alkaline Phosphatase Assay kit; Beyotime Institute of Biotechnology). Subsequently, the mixture was incubated for $10 \mathrm{~min}$ at $37^{\circ} \mathrm{C}$ and a microplate reader was used to measure the absorbance of p-nitrophenol at $405 \mathrm{~nm}$ after termination of the reaction.

Dual luciferase assay. The psiCHECK2 luciferase reporter plasmid (Promega Corporation) was used to clone the synthesized wild-type (WT) and mutant 3'-untranslated region (UTR) of sirt1. The psiCHECK2 plasmid with WT or mutant derivatives and miRNA control or miR-22 mimic were co-transfected into the osteoblasts. Xfect ${ }^{\mathrm{TM}}$ RNA transfection reagent (Takara Biotechnology Co., Ltd.) was used to perform plasmid and miRNA control, as well as miR-22 mimic transfection. Cell lysates were collected $24 \mathrm{~h}$ post-transfection and the dual-luciferase reporter system (Dual-Luciferase ${ }^{\circledR}$ Reporter Assay System; cat. no. E1910; Promega Corporation) was used to measure firefly and Renilla luciferase activity. The ratio of luminescence between the experimental reporter (firefly) to the control reporter (Renilla) was used to reflect the relative luciferase activity of sirt1.

Reverse transcription-quantitative PCR ( $q P C R)$. Total RNA was extracted from the osteoblasts using TRIzol ${ }^{\circledR}$ (Takara Biotechnology Co., Ltd.) and reverse transcribed into cDNA using SuperScript ${ }^{\mathrm{TM}}$ II reverse transcriptase (Thermo Fisher Scientific, Inc.) with a special stem-loop primer for miR-22 and oligodeoxythymidine for mRNAs with the following temperature protocol: $25^{\circ} \mathrm{C}$ for $10 \mathrm{~min} ; 42^{\circ} \mathrm{C}$ for $1 \mathrm{~h}$ and $72^{\circ} \mathrm{C}$ for $10 \mathrm{~min})$. A MiniOpticon real-time PCR detection system (Bio-Rad Laboratories, Inc.) was used to perform qPCR. The reaction solution consisted of $5.0 \mu \mathrm{l}$ diluted cDNA, $0.2 \mu \mathrm{M} / 1$ of each paired primer, 1x Sybr Green qPCR Mix buffer (Toyobo Biotech Co., Ltd.) and 4.9 $\mu \mathrm{l}$ DEPC-treated water. The annealing temperature was set at $58-62^{\circ} \mathrm{C}$ and amplification was set at 40 cycles. The temperature range to detect the melting temperature of the PCR product was set from $60-95^{\circ} \mathrm{C}$. To determine the relative quantitation of gene expression, the comparative $\mathrm{Cq}$ (threshold cycle) method with arithmetic formulae $\left(2^{-\Delta \Delta \mathrm{Cq}}\right)$ was used (25). mRNA levels of sirt1, p53 and p21 were normalized relative to the house-keeping gene $\beta$-actin. The primers used were as follows: Sirt1 (accession number NM_001159589.2) sense strand, 5'-CTGTTTCCT GTGGGATACCTGACT-3' and antisense strand, 5'-ATCGAA CATGGCTTGAGGATCT-3' (26); p53 (accession number NM_001127233.1) sense strand, 5'-AGAGACCGCCGTACA GAAGA-3' and antisense strand, 5'-CTGTAGCATGGGCAT CCTTT-3' (27); p21 (accession number NM_001111099.2) sense strand, 5'-AGCAAAGTGTGCCGTTGT CT-3' and antisense strand, 5'-AGAAATCTGTCAGGCTGGTC-3'; $\beta$-actin (accession number NM_007393.5) sense strand, 5'-AGCCAT GTACGTAGCCATCC-3' and antisense strand, 5'-CTCTCA GCTGTGGTGGTGAA-3' (28). The sequence of miR-22 was AAGCUGCCAGUUGAAGAACUGU (29).

Western blot analysis. Osteoblast proteins were extracted using RIPA buffer including $1 \%$ protease inhibitor cocktail (Thermo Fisher Scientific, Inc.) and BCA assay was used to calculate the protein concentration. Then $30 \mu \mathrm{g}$ proteins per 
A

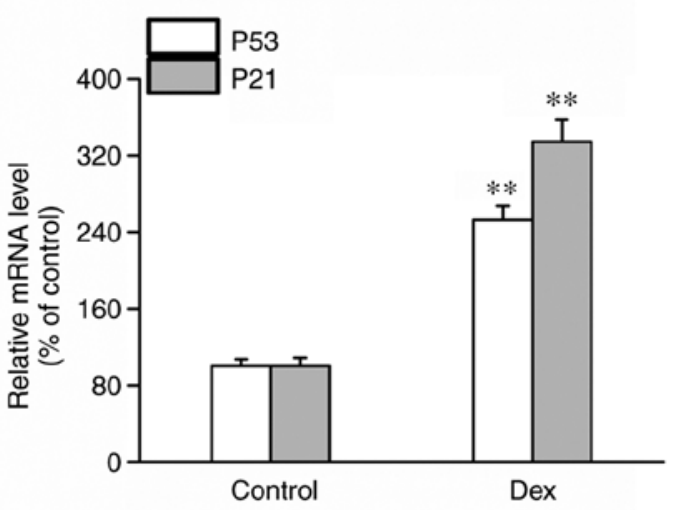

C

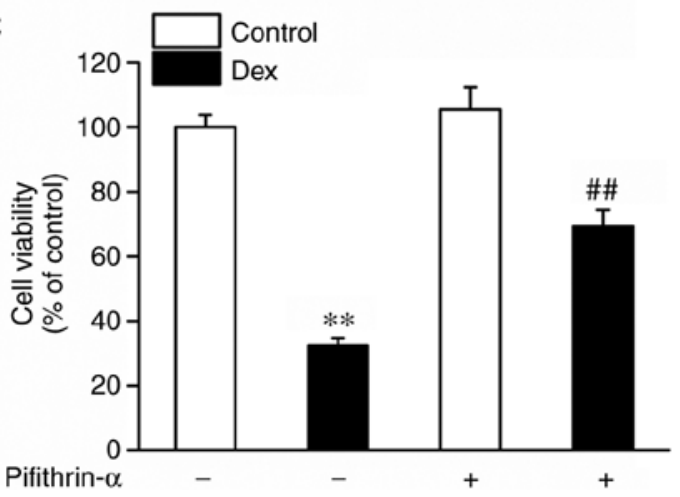

B
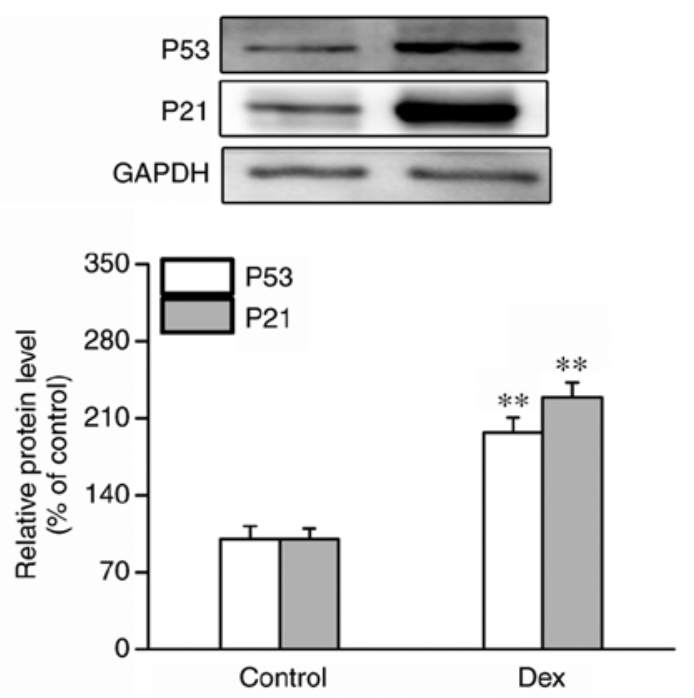

D

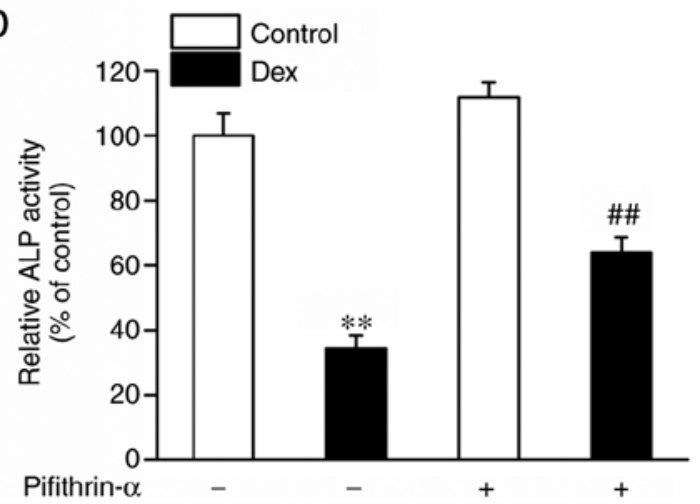

Figure 1. Cell senescence contributed to Dex-induced osteoblast dysfunction. Osteoblasts were exposed to Dex (1 $\mu \mathrm{M})$ for $72 \mathrm{~h}$ and cells treated with an equivalent volume of absolute ethanol $(20 \mu \mathrm{l})$ were used as the control. Reverse transcription-quantitative PCR and western blot analysis were performed to determine p53 and p21 (A) mRNA and (B) protein expression levels in osteoblasts. (C) Cell viability and (D) ALP activity were also measured. Data are presented as the mean $\pm \operatorname{SEM}(\mathrm{n}=4) .{ }^{* *} \mathrm{P}<0.01$ vs. the control. ${ }^{\# \#} \mathrm{P}<0.01$ vs. Dex. Dex, dexamethasone; ALP, alkaline phosphatase.

lane was subjected to SDS-PAGE (10\% gels) to separate, prior to their transfer onto PVDF membranes. After blocking with nonfat dry milk dissolved in TBS containing $0.05 \%$ Tween-20 for 1-2 $\mathrm{h}$ at room temperature, the membranes were incubated with antibodies against sirt1 (Santa Cruz Biotechnology, Inc.; cat. no. sc-74665); p53 (ProteinTech Group, Inc.; cat. no. 10442-1-AP) and p21 (ProteinTech Group, Inc.; cat. no. 28248-1-AP) and GAPDH (Santa Cruz Biotechnology, Inc.; cat. no. sc-137179) overnight at $4^{\circ} \mathrm{C}$ at a dilution of 1:1,000. Subsequently, the membranes were incubated with a secondary horseradish peroxidase-conjugated antibody for 1-2 $\mathrm{h}$ at room temperature at a dilution of 1:2,000, including goat anti-rabbit IgG-HRP (Santa Cruz Biotechnology, Inc.; cat. no. sc-2004) and goat anti-mouse IgG-HRP (Santa Cruz Biotechnology, Inc; cat. no. sc-2005). An enhanced chemiluminescence western blotting detection system (Santa Cruz Biotechnology, Inc.) and a GeneGnome HR scanner (Syngene) were used to visualize the immunoreactive proteins and chemiluminescent signal from the membranes. The expression levels of the proteins of interest were normalized to GAPDH.

Bioinformatics analysis. The TargetScan (http://www.targetscan.org/) and miRanda, (http://www. microrna.org/microrna/home.do) tools were used to perform target prediction. The target genes predicted by TargetScan and miRanda were screened based on their scoring criteria. In the TargetScan algorithm, target genes with a context percentile $<50$ were excluded. In the miRanda algorithm, target genes with Max-Energy >-10 were excluded. The overlapping prediction results were selected as the candidate target genes of miR-22 (30,31).

Statistical analysis. SPSS 16.0 (SPSS, Inc.) was used to perform the data analysis. Data are presented as the mean \pm SEM. Statistical comparisons between two groups were determined using two-tailed paired Student's t-test. One-way or two-way ANOVAs, with Bonferroni's post hoc tests were performed for comparisons among multiple groups. $\mathrm{P}<0.05$ was considered to indicate a statistically significant value.

\section{Results}

Osteoblast senescence contributes to Dex-induced osteoblast dysfunction. Firstly, alterations in the levels of the senescence-associated biomarkers, p53 and p21, in osteoblastic MC3T3-E1 cells exposed to Dex, were measured in the present study. As shown in Fig. 1A and B, the mRNA and protein expression levels of p53 and p21 were significantly 

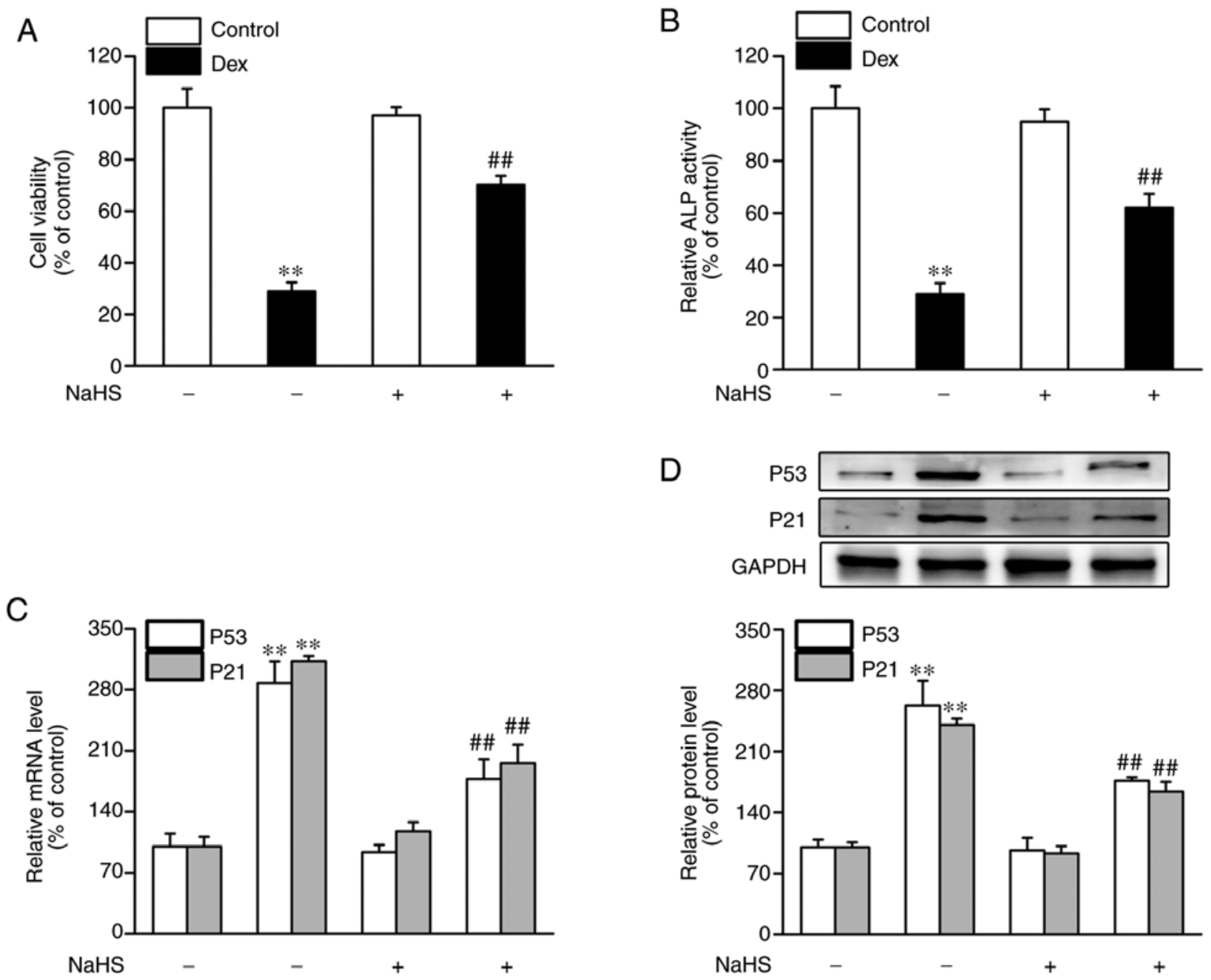

Figure 2. Impact of NaHS on cell senescence and dysfunction in osteoblasts exposed to Dex. Osteoblasts were exposed to Dex (1 $\mu \mathrm{M})$ and NaHS (20 $\mu \mathrm{M})$ for $72 \mathrm{~h}$ and an equivalent volume (20 $\mu \mathrm{l}$ absolute ethanol and $20 \mu \mathrm{l}$ saline solution) of vehicle-treated cells were used as the control. (A) Cell viability and (B) ALP activity, as well as (C) mRNA and (D) protein expression levels of p53 and p21 were measured. Data are presented as mean \pm SEM $(n=4){ }^{* *} \mathrm{P}<0.01$ vs. the control; ${ }^{\# \#} \mathrm{P}<0.01$ vs. Dex. Dex, dexamethasone; ALP, alkaline phosphatase; NaHS, sodium hydrosulfide.

increased in the Dex-treated osteoblasts, suggesting that osteoblast senescence was involved in Dex-induced cell damage. Subsequently, a p53 inhibitor (pifithrin- $\alpha$ ) was used to measure the impact of Dex-induced osteoblast damage. It was found that Dex treatment resulted in a decreased cell viability and ALP activity, with these results being partly reversed by pifithrin- $\alpha$, as shown by the increased cell viability and ALP activity (Fig. 1C and D).

NaHS mitigates Dex-induced osteoblast dysfunction and senescence. Subsequently, the effects of NaHS on osteoblast dysfunction and senescence exposed to Dex were measured. As shown in Fig. 2, compared with control group, cell viability and ALP activity in the Dex-treated group were markedly decreased, whereas the expression of p53 and p21 at the mRNA and protein levels was significantly increased. However, NaHS attenuated Dex-induced osteoblast damage and senescence, as shown by the increased cell viability (Fig. 2A) and the increase in ALP activity (Fig. 2B) in the Dex and NaHS treated group. Additionally, NaHS treatment caused an increase in the mRNA and protein expression levels of p53 and p21 (Fig. 2C and D).
miR-22 mimic blocks the beneficial effects of NaHS on Dex-induced osteoblast dysfunction and senescence. In the present set of experiments, it was found that the miR-22 levels in MC3T3-E1 cells that Dex-treated were significantly increased (Fig. 3A). Subsequently, the present study investigated whether alterations in the miR-22 levels were also associated with the protective effect of NaHS against Dex-induced osteoblast damage and senescence. As shown in Fig. 3A, the increased miR-22 levels in Dex-treated MC3T3-E1 cells was reversed upon administration of NaHS. Moreover, as shown in Fig. S1A, miR-22 mimic transfection resulted in a markable increase expression of miR-22. It was found that the miR-22 mimic blocked the NaHS-induced increases in cell viability and ALP activity that were observed in MC3T3-E1 cells exposed to Dex (Fig. 3B and C). Furthermore, the miR-22 mimic also reversed the NaHS-induced downregulation of p53 and p21 at the mRNA and protein levels in MC3T3-E1 cells subjected to Dex (Fig. 3D and E).

sirtl is the target of miR-22 in osteoblastic MC3T3-E1 cells. As previously mentioned, miRs are able to posttranscriptionally and negatively regulate the expression of target genes (15-17). TargetScan and miRanda were used to 
A

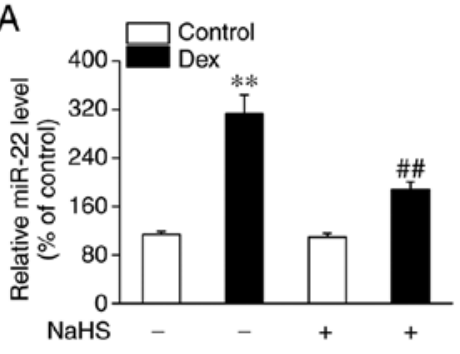

B

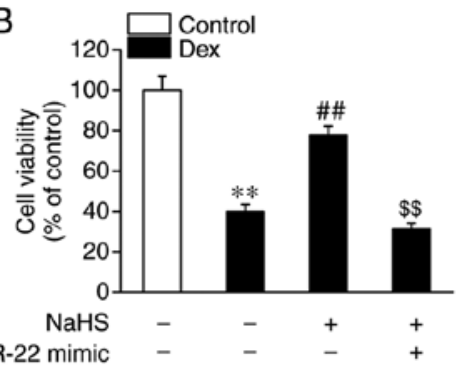

C

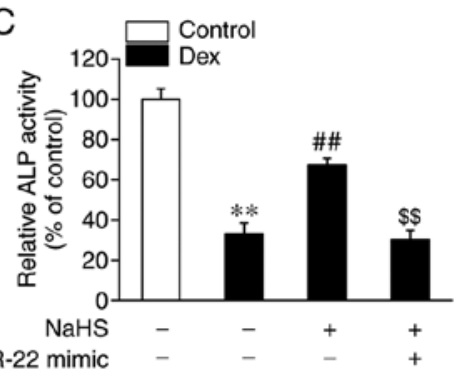

D

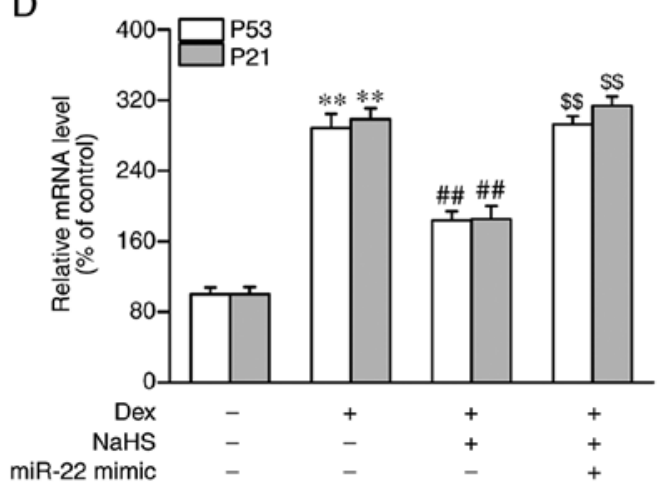

E
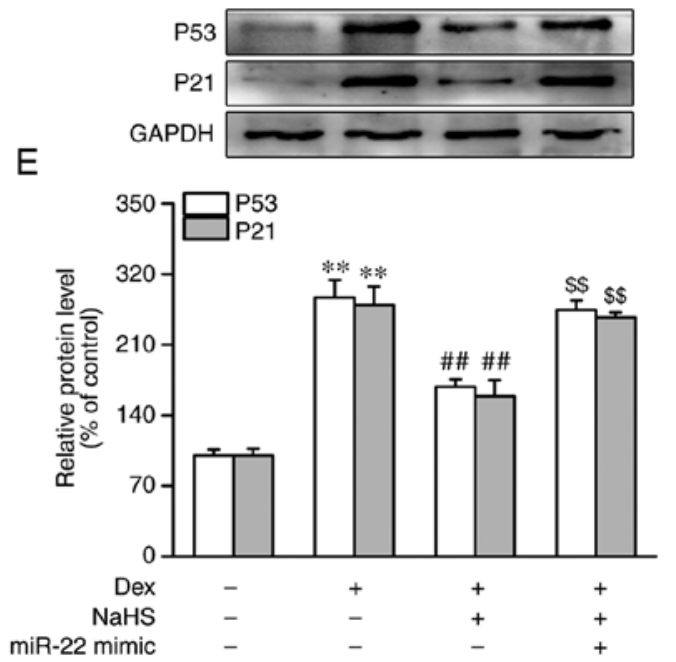

Figure 3. Impact of miR-22 on the protective role of NaHS on Dex-induced osteoblast dysfunction and senescence. Osteoblasts were treated with vehicle; Dex and vehicle; or NaHS, and then subjected to treatment with miR control or miR-22 mimic. (A) Reverse transcription-quantitative PCR was performed to measure the expression levels of miR-22. (B) Cell viability and (C) ALP activity, as well as the (D) mRNA and (E) protein expression levels of p53 and p21 were measured. Data are presented as the mean \pm SEM $(n=4) .{ }^{* *} \mathrm{P}<0.01$ vs. the control + vehicle; ${ }^{\# \#} \mathrm{P}<0.01$ vs. Dex + vehicle; ${ }^{\$ \$} \mathrm{P}<0.01 \mathrm{vs}$. Dex $+\mathrm{NaHS}$. Dex, dexamethasone; ALP, alkaline phosphatase; NaHS, sodium hydrosulfide; miR, microRNA.

A

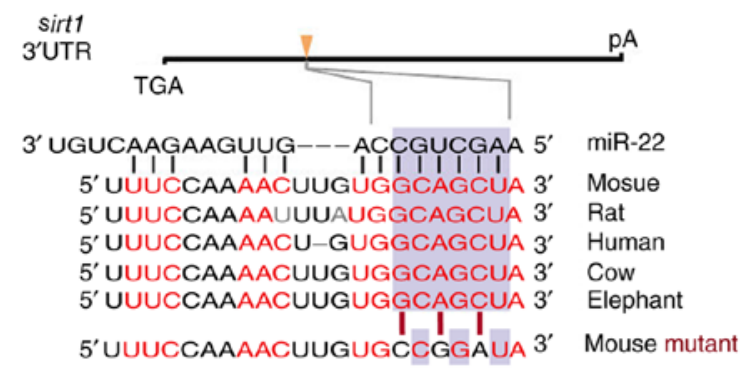

C

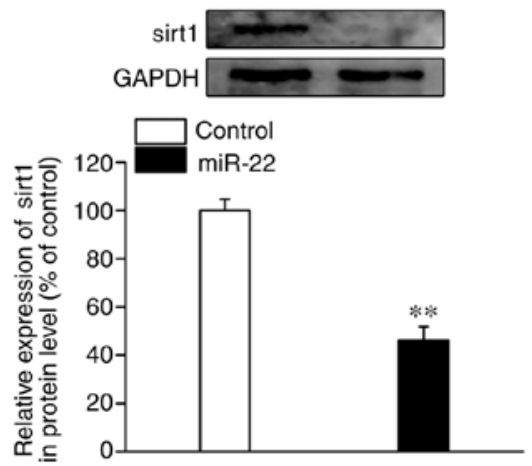

B

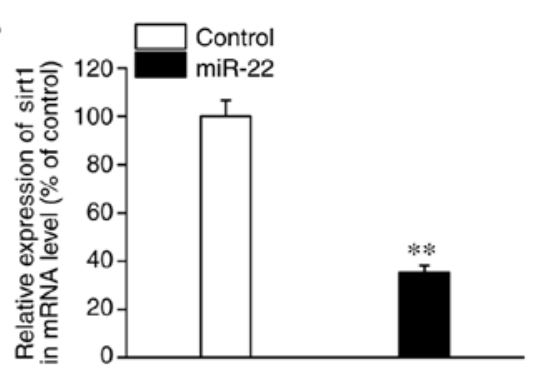

D

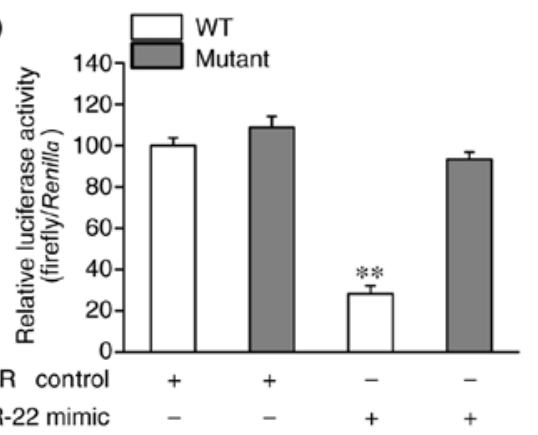

Figure 4. Sirt1 is the target of miR-22 in osteoblastic MC3T3-E1 cells. (A) Sirt1 contains highly conserved miR-22-mRNA interaction motifs within the 3'-UTR. The sequences of the WT and mutant 3'-UTR of sirt1 for the luciferase reporter assay are shown. Osteoblasts were transfected with miR control or miR-22 mimic ( $200 \mathrm{nM}$ ) for $24 \mathrm{~h}$. Reverse transcription-quantitative PCR and western blot analyses were performed to measure the (B) mRNA and (C) protein expression levels of sirt1 in osteoblastic MC3T3-E1 cells. (D) Osteoblasts were co-transfected with $0.4 \mu \mathrm{g}$ WT or mutant sirt1 3'-UTR reporter plasmids in the presence of miR control or miR-22 mimic $(200 \mathrm{nM})$. Luciferase activity was detected using dual luciferase assays after $24 \mathrm{~h}$. Data are presented as the mean $\pm \operatorname{SEM}(\mathrm{n}=4) .{ }^{* *} \mathrm{P}<0.01$ vs. the control. WT, wild-type; 3'-UTR, 3'-untranslated region; sirt1, sirtuin 1; miR, microRNA. 

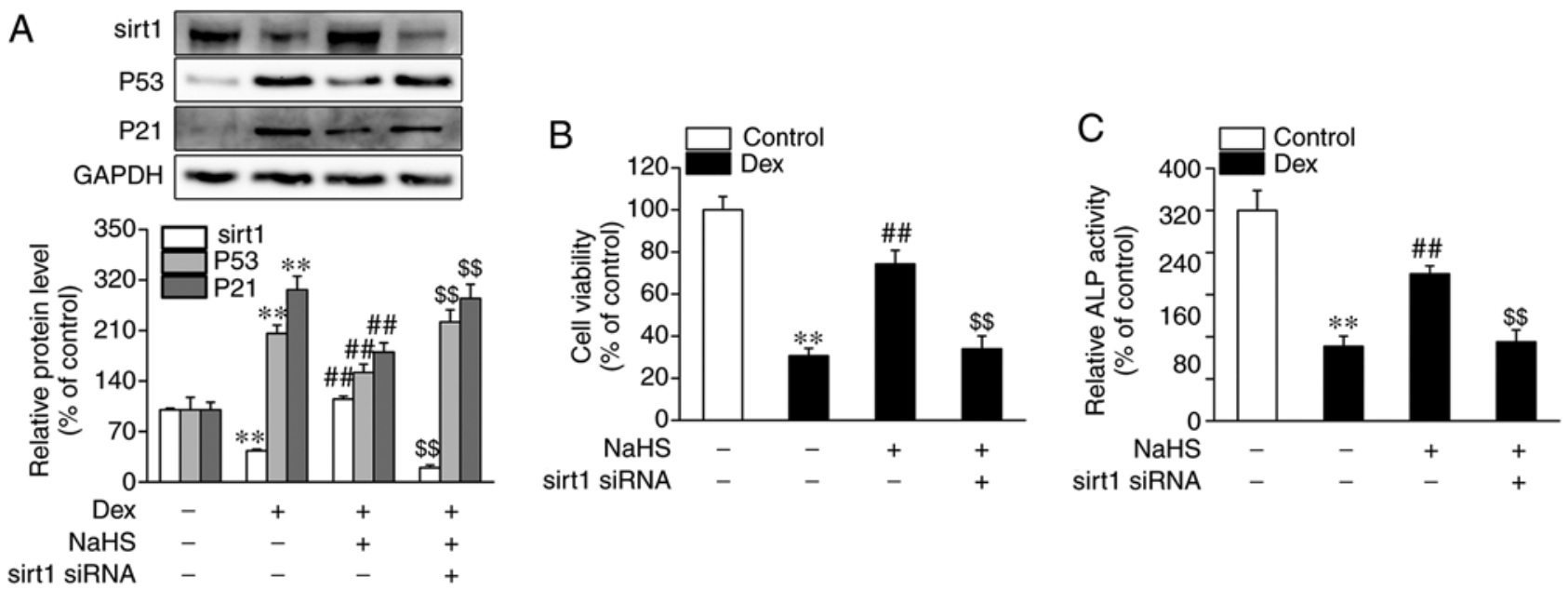

Figure 5. Impact of sirt1 siRNA on the protective role of NaHS against Dex-induced damage and senescence in osteoblastic MC3T3-E1 cells. Osteoblasts were transfected with control siRNA or sirtl siRNA for $24 \mathrm{~h}$, then subjected to treatment with vehicle; Dex and vehicle; or NaHS for $48 \mathrm{~h}$. (A) Cell viability and (B) ALP activity as well as changes in the (C) mRNA and protein expression levels of sirtl were assessed. Data are shown as the mean \pm SEM ( $=4$ ). ${ }^{* *} \mathrm{P}<0.01$ vs. the control; ${ }^{\# \#} \mathrm{P}<0.01$ vs. DEX; ${ }^{\$ \$} \mathrm{P}<0.01$ vs. Dex + sirt1 siRNA. siRNA, small interfering RNA; sirt1, sirtuin 1; NaHS, sodium hydrosulfide; Dex, dexamethasone; ALP, alkaline phosphatase.

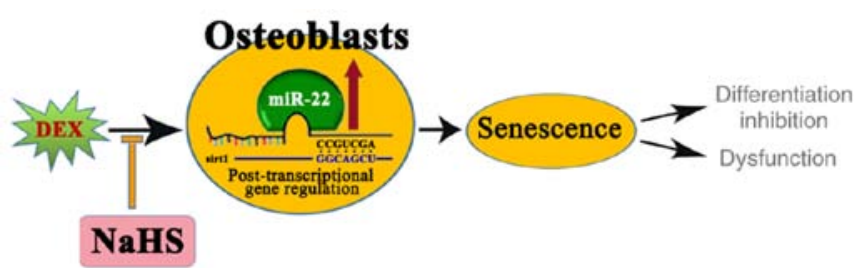

Figure 6. A graphical illustration for the possible mechanisms underlying the protective role of NaHS in Dex-induced osteoblast dysfunction. Dex increased miR-22 expression in osteoblastic MC3T3-E1 cells, which negatively regulated the expression of sirt 1 through post-transcriptional regulation mechanism and then induced cell senescence as well as osteoblast dysfunction and differentiation inhibition. These effects were dramatically attenuated by treatment with the hydrogen sulfide donor, NaHS. NaHS, sodium hydrosulfide; Dex, dexamethasone; miRNA, microRNA.

perform target prediction analysis of miR-22, and sirt1 was found to be an overlapping gene. As shown in Fig. 4A, one evolutionarily conserved sequence of the sirt 1 gene was complementary to miR-22. As shown in Fig. 4B and C, the miR-22 mimic was able to significantly suppress the mRNA and protein expression levels of sirt1. Sirt1 3'-UTR luciferase reporter constructs and miR-22 mimics were transfected into osteoblastic MC3T3-E1 cells to confirm the direct regulatory effect of miR-22 on sirt1 expression. A significant decrease in luciferase activity in the miR-22 mimic group was found compared with the miR control group (Fig. 4D). No decrease in luciferase activity was observed upon co-transfection of miR-22 mimic with the sirt1 3'UTR luciferase reporter construct containing a mutated target sequence. These results suggested that miR-22 could suppress sirt1 expression in osteoblastic MC3T3-E1 cells through binding to response elements in its $3^{\prime}$-UTR.

Silencing of sirtl blocks the protective role of NaHS against Dex-induced osteoblast damage and senescence. As shown in Fig. 5A, the protein expression level of sirtl was significantly decreased in Dex-treated osteoblasts, although this was reversed upon administration of NaHS. Furthermore, the addition of sirtl siRNA led to a marked decrease in mRNA and protein expression levels of sirt1 (Fig. S1B and C). Consequently, whether sirt1 is associated with the beneficial effects of NaHS against Dex-induced osteoblast senescence was investigated. As shown in Fig. 5A, sirt1 siRNA blocked the inhibitory effect of NaHS against Dex-induced osteoblast senescence, as shown by the increased protein levels of p53 and $\mathrm{p} 21$. In addition, sirt1 siRNA also abolished the beneficial effects of NaHS against Dex-induced osteoblast damage, as evidenced by decreased cell viability and ALP activity (Fig. 5B and C).

\section{Discussion}

In the present study, it has been shown that osteoblast senescence may contribute to Dex-induced dysfunction and that a p53 inhibitor (pifithrin- $\alpha$ ) could partly reverse the osteoblast damage induced by Dex, as revealed by increased levels of cell proliferation and ALP activity. Moreover, NaHS mitigated the Dex-induced damage and senescence through targeting the miR-22/sirt1 pathway, as miR-22 mimic and sirt1 siRNA blocked the beneficial effects of NaHS on Dex-induced osteoblast dysfunction and senescence, and sirtl is a target of miR-22.

An accumulating number of studies have suggested that cell senescence exerts a dominant role in a wide variety of diseases, including hepatocarcinogenesis (32), intervertebral disc degeneration (33), diabetic nephropathy (34), diabetic cardiomyopathy (35), Parkinson's disease (36) and Alzheimer's disease (37). Additionally, the crucial role of cell senescence in the development of osteoporosis has also been widely recognized $(38,39)$. Zhang et al $(38)$ revealed that osteoblastic cell senescence may contribute to osteoporosis resulting from estrogen deficiency, and this process is accelerated upon treatment with serum from ovariectomized rats. Khosla et al (39) determined that this may be a novel therapeutic paradigm for restraining, or even reversing, age-associated osteoporosis via targeting cellular senescence. 
It is widely accepted that sirt1 protects osteoblastic cells against multiple unfavorable factors, including hypoxia (40); TiAl6V4 particles and CoCrMo particles (41); hydrogen peroxide (42); and sodium fluoride (43). Interestingly, it is also recognized that sirt1 prevents cell senescence, with sirt1 knockdown inducing cell senescence (44-48). Ota et al (44) demonstrated that overexpression of sirt1 could provide protection against stress-caused endothelial damage through repressing premature senescence of human endothelial cells. $\mathrm{Zu}$ et al (46) also showed that sirtl prevents primary porcine aortic endothelial cell senescence by targeting serine/threonine kinase 11. In the present study, senescence and dysfunction in osteoblastic MC3T3-E1 cells were phenotypically shown to be associated with decreased sirt1 expression levels. Among many factors that negatively regulate gene expression, an accumulating number of studies have provided confirmatory evidence in support of an essential role for miRs (15-17). miR-22 is a widely expressed miR, and bioinformatics analysis and previous studies $(21,22,49)$ have shown that sirt1 is the target of miR-22 in osteoblastic MC3T3-E1 cells. Chen et al (21) demonstrated that miR-22 induces human glioblastoma cell dysfunction through the direct targeting of sirt1, as revealed by suppressed levels of cell proliferation, motility and invasion. Zou et al (22) also revealed that miR-22 could restrict cell growth and metastasis in breast cancer through targeting sirt1 and the miR-22/sirt1 signaling pathway, also proposing that this potentially forms the basis of a novel therapeutic strategy for breast cancer. Another study demonstrated that upregulation of miR-22 contributes to ischemia-reperfusion-induced myocardial damage by directly targeting sirt1, which exerts a critical role in the regulation of mitochondrial function (17). In the present study, it was also shown that upregulation of miR-22 is involved in Dex-induced osteoblastic cell senescence and injury through targeting the regulation of sirt1 expression. However, further research is required to determine whether alteration of the mitochondrial function in Dex-treated osteoblast cells is involved in the underlying mechanism of action.

Previous studies have demonstrated that NaHS, an $\mathrm{H}_{2} \mathrm{~S}$ donor, exerts a protective role in multiple diseases, including chronic restrain stress (CRS)-induced cognitive damage (50), cerebral IR injury (51), chronic kidney disease $(52,53)$ and high pulmonary blood flow-induced pulmonary artery collagen remodeling (54). Interestingly, Li et al (50) showed that $\mathrm{H}_{2} \mathrm{~S}$ mitigates $\mathrm{CRS}$-induced cognitive damage and hippocampal impairment through the upregulation of sirt1. A growing number of studies have shown that $\mathrm{H}_{2} \mathrm{~S}$ may protect a variety of cell types from adverse factors by delaying cell senescence (55-57). Zheng et al (57) demonstrated that NaHS delays cellular senescence of human umbilical vascular endothelial cells through upregulating sirt1 expression. In agreement with these results, the results of the present study have also demonstrated that NaHS mitigated Dex-induced osteoblastic cell senescence and impairment through the upregulation of sirt1 expression.

A limitation of the present study was that the results were gained in vitro. Further in vivo studies would be beneficial to confirm the present findings. Interestingly, Ma et al (58) demonstrated that exogenous $\mathrm{H}_{2} \mathrm{~S}$ attenuates Dex-induced inhibition of osteoblast proliferation and osteogenic differentiation by activating the $\mathrm{Wnt} / \beta$-catenin signaling pathway.
Additionally, Xia et al (59) indicated that activation of the Wnt/ $\beta$-catenin signaling pathway is negatively related to cell senescence. As such, it was speculated that exogenous $\mathrm{H}_{2} \mathrm{~S}$ alleviates Dex-induced osteoblast dysfunction through inhibition of senescence. Furthermore, future studies should investigate the mechanism of action behind how Dex treatment leads to increased expression levels of miR-22, as well as the other targets of miR-22 in Dex-treated osteoblasts.

As summarized in Fig. 6, the present study demonstrated that NaHS prevented Dex-induced osteoblastic MC3T3-E1 cell senescence and damage through targeting the miR-22/sirtl pathway.

\section{Acknowledgements}

Not applicable.

\section{Funding}

The present study was supported by the key incubation project of Li Peng from the Science and Technology Department of Ningxia Medical University, Key Incubation Project.

\section{Availability of data and materials}

The datasets used and/or analyzed during the current study are available from the corresponding author on reasonable request.

\section{Author's contributions}

ZDL was responsible for study design and manuscript preparation. PL performed manuscript preparation, data collection and statistical analysis. WWM contributed to data interpretation. The literature search and dual luciferase assay were performed by SZ, LZ and ZRC. All authors read and approved the final manuscript.

\section{Ethics approval and consent to participate}

Not applicable.

\section{Patient consent for publication}

Not applicable.

\section{Competing interests}

The authors declare that they have no competing interests.

\section{References}

1. van Staa TP, Leufkens HG, Abenhaim L, Begaud B, Zhang B and Cooper C: Use of oral corticosteroids in the United Kingdom. QMJ 93: 105-111, 2000

2. Fardet L, Petersen I and Nazareth I: Prevalence of long-term oral glucocorticoid prescriptions in the UK over the past 20 years. Rheumatology (Oxford) 50: 1982-1990, 2011.

3. Overman RA, Yeh JY and Deal CL: Prevalence of oral glucocorticoid usage in the United States: A general population perspective. Arthritis Care Res (Hoboken) 65: 294-298, 2013.

4. Silverman S, Curtis J, Saag K, Flahive J, Adachi J, Anderson F, Chapurlat R, Cooper C, Diez-Perez A, Greenspan S, et al: International management of bone health in glucocorticoidexposed individuals in the observational GLOW study. Osteoporos Int 26: 419-420, 2015 . 
5. Ventura A, Brunetti G, Colucci S, Oranger A, Ladisa F, Cavallo L, Grano M and Faienza MF: Glucocorticoid-induced osteoporosis in children with 21-hydroxylase deficiency. Biomed Res Int 2013: 250462, 2013.

6. Delany AM, Dong Y and Canalis E: Mechanisms of glucocorticoid action in bone cells. J Cell Biochem 56: 295-302, 1994.

7. O'Brien CA, Jia D, Plotkin LI, Bellido T, Powers CC, Stewart SA, Manolagas SC and Weinstein RS: Glucocorticoids act directly on osteoblasts and osteocytes to induce their apoptosis and reduce bone formation and strength. Endocrinology 145: 1835-1841, 2004.

8. Burton DG and Krizhanovsky V: Physiological and pathological consequences of cellular senescence. Cell Mol Life Sci 71: 4373-4386, 2014.

9. Wu G, Xu R, Zhang P, Xiao T, Fu Y, Zhang Y, Du Y, Ye J, Cheng J and Jiang H: Estrogen regulates stemness and senescence of bone marrow stromal cells to prevent osteoporosis via ER $\beta$-SATB2 pathway. J Cell Physiol 233: 4194-4204, 2018.

10. Elrod JW, Calvert JW, Morrison J, Doeller JE, Kraus DW, Tao L, Jiao X, Scalia R, Kiss L, Szabo C, et al: Hydrogen sulfide attenuates myocardial ischemia-reperfusion injury by preservation of mitochondrial function. Proc Natl Acad Sci USA 104: 15560-15565, 2007.

11. Kimura $\mathrm{Y}$ and Kimura $\mathrm{H}$ : Hydrogen sulfide protects neurons from oxidative stress. FASEB J 18: 1165-1167, 2004.

12. Esechie A, Kiss L, Olah G, Horváth EM, Hawkins H, Szabo C and Traber DL: Protective effect of hydrogen sulfide in a murine model of acute lung injury induced by combined burn and smoke inhalation. Clin Sci (Lond) 115: 91-97, 2008.

13. Xu ZS, Wang XY, Xiao DM, Hu LF, Lu M, Wu ZY and Bian JS Hydrogen sulfide protects MC3T3-E1 osteoblastic cells against $\mathrm{H} 2 \mathrm{O} 2$-induced oxidative damage-implications for the treatment of osteoporosis. Free Radic Biol Med 50: 1314-1323, 2011.

14. Yang M,Huang Y, Chen J, Chen YL, Ma JJ and Shi PH: Activation of AMPK participates hydrogen sulfide-induced cyto-protective effect against dexamethasone in osteoblastic MC3T3-E1 cells. Biochem Biophys Res Commun 454: 42-47, 2014.

15. Banzhaf-Strathmann J, Benito E, May S, ArzbergerT, Tahirovic S, Kretzschmar H, Fischer A and Edbauer D: MicroRNA-125b induces tau hyperphosphorylation and cognitive deficits in Alzheimer's disease. EMBO J 33: 1667-1680, 2014.

16. Harada M, Luo X, Murohara T, Yang B, Dobrev D and Nattel S: MicroRNA regulation and cardiac calcium signaling: Role in cardiac disease and therapeutic potential. Circ Res 114: 689-705, 2014.

17. Du JK, Cong BH, Yu Q, Wang H, Wang L, Wang CN, Tang XL, Lu JQ, Zhu XY and Ni X: Upregulation of microRNA-22 contributes to myocardial ischemia-reperfusion injury by interfering with the mitochondrial function. Free Radic Biol Med 96: 406-417, 2016

18. Shi C, Qi J, Huang P, Jiang M, Zhou Q, Zhou H, Kang H, Qian N, Yang Q, Guo L and Deng L: MicroRNA-17/20a inhibits glucocorticoid-induced osteoclast differentiation and function through targeting RANKL expression in osteoblast cells. Bone 68: 67-75, 2014.

19. Zhang Y, Gao Y, Cai L, Li F, Lou Y, Xu N, Kang Y and Yang H: MicroRNA-221 is involved in the regulation of osteoporosis through regulates RUNX2 protein expression and osteoblast differentiation. Am J Transl Res 9: 126-135, 2017.

20. Liang WC, Fu WM, Wang YB, Sun YX, Xu LL, Wong CW, Chan KM, Li G, Waye MM and Zhang JF: H19 activates Wnt signaling and promotes osteoblast differentiation by functioning as a competing endogenous RNA. Sci Rep 6: 20121, 2016.

21. Chen H, Lu Q, Fei X, Shen L, Jiang D and Dai D: miR-22 inhibits the proliferation, motility, and invasion of human glioblastoma cells by directly targeting SIRT1. Tumour Biol 37: 6761-6768, 2016.

22. Zou Q, Tang Q, Pan Y, Wang X, Dong X, Liang Z and Huang D: MicroRNA-22 inhibits cell growth and metastasis in breast cancer via targeting of SIRT1. Exp Ther Med 14: 1009-1016, 2017.

23. Tang YH, Yue ZS, Li GS, Zeng LR, Xin DW, Hu ZQ and Xu CD: Effect of $\beta$-ecdysterone on glucocorticoid-induced apoptosis and autophagy in osteoblasts. Mol Med Rep 17: 158-164, 2018.

24. Bowers GN Jr and McComb RB: A continuous spectrophotometric method for measuring the activity of serum alkaline phosphatase. Clin Chem 12: 70-89, 1966.

25. Livak KJ and Schmittgen TD: Analysis of relative gene expression data using real-time quantitative PCR and the 2(-Delta Delta C(T)) method. Methods 25: 402-408, 2001.
26. Zhang HH, Ma XJ, Wu LN, Zhao YY, Zhang PY, Zhang YH, Shao MW, Liu F, Li F and Qin GJ: SIRT1 attenuates high glucose-induced insulin resistance via reducing mitochondrial dysfunction in skeletal muscle cells. Exp Biol Med (Maywood) 240: 557-565, 2015.

27. Cho SJ, Rossi A, Jung YS, Yan W, Liu G, Zhang J, Zhang M and Chen X: Ninjurin1, a target of p53, regulates p53 expression and p53-dependent cell survival, senescence, and radiation-induced mortality. Proc Natl Acad Sci USA 110: 9362-9367, 2013

28. Lee CJ, Kim HT, Song KW, Kim SS, Park HH and Yoon YD: Ovarian expression of p53 and p21 apoptosis regulators in gamma-irradiated mice. Mol Reprod Dev 75: 383-391, 2008.

29. Lagos-Quintana M, Rauhut R, Yalcin A, Meyer J, Lendeckel W and Tuschl T: Identification of tissue-specific microRNAs from mouse. Curr Biol 12: 735-739, 2002.

30. Meng SS, Wang H, Xue DB and Zhang WH: Screening and validation of differentially expressed extracellular miRNAs in acute pancreatitis. Mol Med Rep 16: 6412-6418, 2017.

31. Sun F, Yang X, Jin Y, Chen L, Wang L, Shi M, Zhan C, Shi Y and Wang Q: Bioinformatics analyses of the differences between lung adenocarcinoma and squamous cell carcinoma using The Cancer Genome Atlas expression data. Mol Med Rep 16: 609-616, 2017.

32. Mossanen JC, Kohlhepp M, Wehr A, Krenkel O, Liepelt A, Roeth AA, Möckel D, Heymann F, Lammers T, Gassler N, et al: CXCR6 inhibits hepatocarcinogenesis by promoting natural killer T- and $\mathrm{CD}^{+}{ }^{+} \mathrm{T}$-cell-dependent control of senescence. Gastroenterology 156: 1877-1889.e4, 2019.

33. Chen J, Xie JJ, Jin MY, Gu YT, Wu CC, Guo WJ, Yan YZ, Zhang ZJ, Wang JL, Zhang XL, et al: Sirt6 overexpression suppresses senescence and apoptosis of nucleus pulposus cells by inducing autophagy in a model of intervertebral disc degeneration. Cell Death Dis 9: 56, 2018.

34. Chen K, Dai H, Yuan J, Chen J, Lin L, Zhang W, Wang L, Zhang J, Li K and He Y: Optineurin-mediated mitophagy protects renal tubular epithelial cells against accelerated senescence in diabetic nephropathy. Cell Death Dis 9: 105, 2018.

35. Gu J, Wang S, Guo H, Tan Y, Liang Y, Feng A, Liu Q, Damodaran C, Zhang Z, Keller BB, et al: Inhibition of p53 prevents diabetic cardiomyopathy by preventing early-stage apoptosis and cell senescence, reduced glycolysis, and impaired angiogenesis. Cell Death Dis 9: 82, 2018.

36. Williams-Gray CH, Wijeyekoon RS, Scott KM, Hayat S, Barker RA and Jones JL: Abnormalities of age-related T cell senescence in Parkinson's disease. J Neuroinflammation 15: 166, 2018.

37. Virgili J, Lebbadi M, Tremblay C, St-Amour I, Pierrisnard C, Faucher-Genest A, Emond V, Julien C and Calon F: Characterization of a 3xTg-AD mouse model of Alzheimer's disease with the senescence accelerated mouse prone 8 (SAMP8) background. Synapse 72, 2018.

38. Zhang J, Lazarenko OP, Blackburn ML, Badger TM, Ronis MJ and Chen JR: Blueberry consumption prevents loss of collagen in bone matrix and inhibits senescence pathways in osteoblastic cells. Age (Dordr) 35: 807-820, 2013.

39. Khosla S, Farr JN and Kirkland JL: Inhibiting cellular senescence: A new therapeutic paradigm for age-related osteoporosis. J Clin Endocrinol Metab 103: 1282-1290, 2018.

40. Zhou L, Wang SI, Moon YJ, Kim KM, Lee KB, Park BH, Jang KY and Kim JR: Overexpression of SIRT1 prevents hypoxia-induced apoptosis in osteoblast cells. Mol Med Rep 16: 2969-2975, 2017.

41. Deng Z, Wang Z, Jin J, Wang Y, Bao N, Gao Q and Zhao J: SIRT1 protects osteoblasts against particle-induced inflammatory responses and apoptosis in aseptic prosthesis loosening. Acta Biomater 49: 541-554, 2017.

42. He N, Zhu X, He W, Zhao S, Zhao W and Zhu C: Resveratrol inhibits the hydrogen dioxide-induced apoptosis via Sirt 1 activation in osteoblast cells. Biosci Biotechnol Biochem 79: 1779-1786, 2015

43. Gu X, Han D, Chen W, Zhang L, Lin Q, Gao J, Fanning S and Han B: SIRT1-mediated FoxOs pathways protect against apoptosis by promoting autophagy in osteoblast-like MC3T3-E1 cells exposed to sodium fluoride. Oncotarget 7: 65218-65230, 2016.

44. Ota H, Akishita M, Eto M, Iijima K, Kaneki M and Ouchi Y: Sirt1 modulates premature senescence-like phenotype in human endothelial cells. J Mol Cell Cardiol 43: 571-579, 2007.

45. Ota H, Tokunaga E, Chang K, Hikasa M, Iijima K, Eto M, Kozaki K, Akishita M, Ouchi Y and Kaneki M: Sirt1 inhibitor, Sirtinol, induces senescence-like growth arrest with attenuated Ras-MAPK signaling in human cancer cells. Oncogene 25: $176-185,2006$. 
46. Zu Y, Liu L, Lee MY, Xu C, Liang Y, Man RY, Vanhoutte PM and Wang Y: SIRT1 promotes proliferation and prevents senescence through targeting LKB1 in primary porcine aortic endothelial cells. Circ Res 106: 1384-1393, 2010.

47. Huang J, Gan Q, Han L, Li J, Zhang H, Sun Y, Zhang Z and Tong T: SIRT1 overexpression antagonizes cellular senescence with activated ERK/S6k1 signaling in human diploid fibroblasts. PLoS One 3: e1710, 2008.

48. Zhao G, Cui J, Zhang JG, Qin Q, Chen Q, Yin T, Deng SC, Liu Y, Liu L, Wang B, et al: SIRT1 RNAi knockdown induces apoptosis and senescence, inhibits invasion and enhances chemosensitivity in pancreatic cancer cells. Gene Ther 18: 920-928, 2011.

49. Ming GF, Wu K, Hu K, Chen Y and Xiao J: NAMPT regulates senescence, proliferation, and migration of endothelial progenitor cells through the SIRT1 AS 1ncRNA/miR-22/SIRT1 pathway. Biochem Biophys Res Commun 478: 1382-1388, 2016.

50. Li XN, Chen L, Luo B, Li X, Wang CY, Zou W, Zhang P, You Y and Tang XQ: Hydrogen sulfide attenuates chronic restrain stress-induced cognitive impairment by upreglulation of Sirt1 in hippocampus. Oncotarget 8: 100396-100410, 2017.

51. Jiang WW, Huang BS, Han Y, Deng LH and Wu LX: Sodium hydrosulfide attenuates cerebral ischemia/reperfusion injury by suppressing overactivated autophagy in rats. FEBS Open Bio 7: $1686-1695,2017$

52. Askari H, Seifi B, Kadkhodaee M, Sanadgol N, Elshiekh M, Ranjbaran M and Ahghari P: Protective effects of hydrogen sulfide on chronic kidney disease by reducing oxidative stress, inflammation and apoptosis. EXCLI J 17: 14-23, 2018.

53. Wu D, Luo N, Wang L, Zhao Z, Bu H, Xu G, Yan Y, Che X, Jiao Z, Zhao T, et al: Hydrogen sulfide ameliorates chronic renal failure in rats by inhibiting apoptosis and inflammation through ROS/MAPK and NF- $\mathrm{B}$ signaling pathways. Sci Rep 7: 455, 2017.
54. Li X, Du J, Jin H, Geng B and Tang C: Sodium hydrosulfide alleviates pulmonary artery collagen remodeling in rats with high pulmonary blood flow. Heart Vessels 23: 409-419, 2008

55. Yang G, Zhao K, Ju Y, Mani S, Cao Q, Puukila S, Khaper N, Wu L and Wang R: Hydrogen sulfide protects against cellular senescence via S-sulfhydration of Keap1 and activation of Nrf2. Antioxid Redox Signal 18: 1906-1919, 2013.

56. Wang WJ, Cai GY, Ning YC, Cui J, Hong Q, Bai XY, Xu XM, $\mathrm{Bu}$ R, Sun XF and Chen XM: Hydrogen sulfide mediates the protection of dietary restriction against renal senescence in aged F344 rats. Sci Rep 6: 30292, 2016.

57. Zheng M, Qiao W, Cui J, Liu L, Liu H, Wang Z and Yan C: Hydrogen sulfide delays nicotinamide-induced premature senescence via upregulation of SIRT1 in human umbilical vein endothelial cells. Mol Cell Biochem 393: 59-67, 2014.

58. Ma J, Shi C, Liu Z, Han B, Guo L, Zhu L and Ye T: Hydrogen sulfide is a novel regulator implicated in glucocorticoidsinhibited bone formation. Aging (Albany NY) 11: 7537-7552, 2019.

59. Xia W, Zhuang L, Deng $X$ and Hou M: Long noncoding RNA-p21 modulates cellular senescence via the Wnt/ $\beta$-catenin signaling pathway in mesenchymal stem cells. Mol Med Rep 16: 7039-7047, 2017.

(i) (2) This work is licensed under a Creative Commons Attribution-NonCommercial-NoDerivatives 4.0 International (CC BY-NC-ND 4.0) License. 